Article

\title{
Phylogeny of Anopheles (Kerteszia) (Diptera: Culicidae) Using Mitochondrial Genes
}

\author{
Karin Kirchgatter ${ }^{1, * \mathbb{C}}$, Lilian de Oliveira Guimarães ${ }^{1}$, Henrry Hugo Yañez Trujillano ${ }^{2} \mathbb{D}$, \\ Fernando Rafael Arias ${ }^{3}{ }^{(0}$, Abraham Germán Cáceres ${ }^{4,5}{ }^{\circledR}$, Ana Maria Ribeiro de Castro Duarte ${ }^{1}$, \\ Rosely dos Santos Malafronte ${ }^{6}$, Rosa Maria Tubaki ${ }^{7}$ and Maria Anice Mureb Sallum ${ }^{8}$ (D) \\ 1 Laboratório de Bioquímica e Biologia Molecular, Superintendência de Controle de Endemias/Instituto \\ de Medicina Tropical de São Paulo, Universidade de São Paulo, SP 01027-000, Brazil; \\ lilianguima@gmail.com (L.d.O.G.); amrcd2@gmail.com (A.M.R.d.C.D.) \\ 2 Area de Vigilancia y Control Vectorial, Dirección de Salud Ambiental, Dirección Ejecutiva de Salud \\ Ambiental, Dirección Regional de Salud Cusco, Cusco 08003, Peru; henrryhugo@yahoo.com \\ 3 Laboratorio de Referencia Regional de Salud Pública, Dirección Regional de Salud Puno, Puno 21002, Peru; \\ fariasvaldivia@hotmail.com \\ 4 Seccion de Entomología, Instituto de Medicina Tropical “Daniel A. Carrión” y Departamento Académico \\ de Microbiología Médica, Facultad de Medicina, Universidad Nacional Mayor de San Marcos, \\ Lima 15081, Peru; acaceres31@hotmail.com \\ 5 Laboratorio de Entomologia, Instituto Nacional de Salud, Lima 15064, Peru \\ 6 Laboratório de Protozoologia, Instituto de Medicina Tropical de São Paulo, Universidade de São Paulo, \\ São Paulo, SP 05403-000, Brazil; rmalafronte@usp.br \\ 7 Laboratório de Entomologia Médica, Superintendência de Controle de Endemias, São Paulo, \\ SP 01027-000, Brazil; tubaki.rm@gmail.com \\ 8 Departamento de Epidemiologia, Faculdade de Saúde Pública, Universidade de São Paulo, São Paulo, \\ SP 01246-904, Brazil; masallum@usp.br \\ * Correspondence: karink@usp.br
}

Received: 3 May 2020; Accepted: 21 May 2020; Published: 24 May 2020

\begin{abstract}
Identification of mosquito species is necessary for determining the entomological components of malaria transmission, but it can be difficult in morphologically similar species. DNA sequences are largely used as an additional tool for species recognition, including those that belong to species complexes. Kerteszia mosquitoes are vectors of human and simian malaria in the Neotropical Region, but there are few DNA sequences of Kerteszia species in public databases. In order to provide relevant information about diversity and improve knowledge in taxonomy of Kerteszia species in Peru, we sequenced part of the mitochondrial genome, including the cytochrome c oxidase I (COI) barcode region. Phylogenetic analyses structured all species of mosquitoes collected in Peru into a single clade, separate from the Brazilian species. The Peruvian clade was composed of two lineages, encompassing sequences from Anopheles (Kerteszia) boliviensis and Anopheles (Kerteszia) pholidotus. An. pholidotus sequences were recorded for the first time in Peru, whereas An. boliviensis sequences were for the first time published in the GenBank database. Sequences generated from specimens morphologically identified as Anopheles (Kerteszia) cruzii clustered into three separate clades according to the collection localities of Serra do Mar, Serra da Mantiqueira, and Serra da Cantareira, confirming An. cruzii as a species complex, composed of at least three putative species.
\end{abstract}

Keywords: mosquitoes; malaria; Kerteszia; Peru; population genetics; barcoding

\section{Introduction}

Malaria is a mosquito-borne tropical disease that remains an important public health problem in some tropical and subtropical countries. In South America, the majority of cases occur in areas of the 
Amazon rainforest, and are mainly concentrated in Venezuela, Brazil, and Peru, which registered 30\%, $24 \%$, and $19 \%$ of the American malaria cases in 2015, respectively [1].

In Peru, malaria transmission occurs throughout the year in rural areas below $2000 \mathrm{~m}$ altitude, with the highest risk in the department of Loreto in the Amazon Region, which registered $96 \%$ of the 55,218 cases reported in the country in 2017 [2]. In 2017, the department of Cusco, where mosquitoes from this study were collected, registered an increase of $41 \%$ in the malaria cases in relation to 2016, being the fifth department in Peru in number of cases [2]. In Lima and areas at high altitude (including the Inca Trail, Machu Picchu, and Lake Titicaca) the malaria risk is low. In 2019, there was a decrease in cases of malaria, with 56\% fewer reported cases compared to the same period in 2018. However, an outbreak of 34 cases of malaria due to Plasmodium vivax was reported in Tumbes Department, in the northern part of the country, which included 22 autochthonous cases and 12 cases imported from Venezuela; this outbreak highlights the risk of re-introduction of malaria in a historically endemic area where transmission had been interrupted in recent years [3].

Plasmodium vivax is responsible for $80 \%$ of the Peruvian malaria cases [4]. However, as in other endemic countries, the incidence of Plasmodium malariae may be underestimated due to difficulties in microscopic differentiation from P. vivax, and its presence in coinfections. Plasmodium malariae has been identified in the vast majority of blood samples from Amerindian ethnic groups from Peru, including coinfection with P. vivax [5]. In areas of the Peruvian Amazon, the primary vector is Anopheles darlingi. This species accounts for $>85 \%$ of the anopheline mosquitoes that were observed to be blood-feeding on humans, and it is responsible for malaria transmission, particularly in frontier areas [6-9]. In Iquitos, Peruvian Amazon, a high proportion of An. darlingi was found blood-feeding on humans and avians, showing the vector a generalist mosquito [10].

In Brazil, 99\% of malaria cases registered are from the Amazon Region, where An. darlingi is the primary vector, and other species can participate as secondary vectors in specific areas [11]. Outside the Amazon region, malaria endemic areas are mainly related to the Atlantic forest biome where Kerteszia species are associated with phytotelma habitats, such as those in the Bromeliaceae family, where they use the axils as a larval habitat [12]. In the Atlantic Forest, Plasmodium infection is usually asymptomatic, caused by sub-patent levels of P. vivax or P. malariae [13], and Anopheles (Kerteszia) cruzii and Anopheles (Kerteszia) bellator are their vectors, with An. cruzii involved in the transmission of both human and simian malaria [14-17].

Anopheles species that use bromeliads phytotelmata as larval habitats have been recognized as a distinct group by Knab (1913). The relationship between the subgenera Kerteszia and Nyssorhynchus has been hypothesized by Root (1922). Following this, Christophers (1924) and Edwards (1932) included Kerteszia in the subgenus Nyssorhynchus, but Dyar (1928) and Komp (1937) treat them as a distinct subgenera [18]. Recently, Foster et al. [19] proposed Kertezia as a genus on the basis of the robust results of mitogenome phylogeny of the subfamily Anophelinae. There are 12 valid Kerteszia species: Anopheles (Kerteszia) auyantepuiensis, Anopheles (Kerteszia) bambusicolus, Anopheles (Kerteszia) bellator, Anopheles (Kerteszia) boliviensis, Anopheles (Kerteszia) cruzii, Anopheles (Kerteszia) gonzalezrinconesi, Anopheles (Kerteszia) homunculus, Anopheles (Kerteszia) laneanus, Anopheles (Kerteszia) lepidotus, Anopheles (Kerteszia) neivai, Anopheles (Kerteszia) pholidotus, and Anopheles (Kerteszia) rollai [20].

Kerteszia mosquitoes are widely distributed from Mexico to southern Brazil [18,21,22]. In Peru, there are records of An. bambusicolus, An. boliviensis, An. cruzii, An. homunculus, An. laneanus, An. lepidotus, An. neivai, and An. pholidotus (ref. in [17]). In Brazil, the occurrences of An. bambusicolus (in areas of the interior of the Atlantic Forest), An. bellator, An. cruzii and An. homunculus (in coastal and mountainous areas of the Serra do Mar in the Atlantic Forest Region), An. laneanus (in areas of Serra da Mantiqueira), and An. neivai (in the Amazon Region) have been registered (ref. in [17]). An. boliviensis was registered in Brazil [23] but the absence of recent records makes its identification questionable [20].

Anopheles homunculus, An. cruzii, An. bellator, and An. laneanus form a well-defined species group, which is always easily separated from the other Kerteszia species [18]. Anopheles homunculus and An. cruzii are morphologically similar on the basis of the external characteristics of the females. 
However, they can be easily identified by characteristics of male genitalia, as well as fourth-instar larva and pupa [24,25]. Anopheles cruzii has been demonstrated to be a species complex on the basis of cytological differences on the banding patterns of polythene chromosomes [26]. Recently, Oliveira et al. [27], using the mitogenome, showed that the specimens from Serra da Cantareira, São Paulo, Brazil, may belong to distinct lineage that is sister to An. cruzii.

Differences in the ecology and behavior of species can affect the transmission of malaria and the success of the methods used in the control, such as insecticides and insecticide-impregnated bed nets [28]. Therefore, the characterization of species composition of a local population for the design of vector control projects is of vital importance. DNA-based methods have been widely used for species identification. Mitochondrial genes have been broadly employed in studies focusing on taxonomy and phylogeny of the Culicidae and Anophelinae subfamilies. The cytochrome coxidase subunit I (COI) gene of the mitogenome has been largely used to identify taxonomic groups at species level $[29,30]$. There are COI sequences available in the GenBank and BOLD databases, including sequences generated for the Mosquito Barcoding Initiative (MBI) (http://barcodeoflife.ning.com/group/mosquitobarcoding).

In the current study, we initially used in the phylogenetic analysis a fragment of mitochondrial cytochrome c oxidase I (COI) that represents the barcode region [31,32]. The aim was to analyze the haplotype diversity and verify the genetic variability of specimens collected in Peru. This mitochondrial region is the main source of reference sequences, and has been successfully used to identify mosquitoes $[25,29,33,34]$ and to evaluate intraspecific variability, especially in Neotropical Anopheles [35-37]. This analysis included mosquitoes from Peru, which were previously identified as An. cruzii, An. boliviensis, An. Laneanus, and An. lepidotus using morphological characteristics. Lastly, we generated two more partial sequences (another COI region and ND4, NADH dehydrogenase subunit 4 gene), totalizing $10 \%$ of the whole mtDNA (mitochondrial DNA) $(\approx 1500 \mathrm{bp}$ ), and compared with reference sequences from a previously published complete mitochondrial DNA genome, to verify whether An. cruzii is a species complex.

\section{Materials and Methods}

\subsection{Mosquito Sampling and Handling}

Females were collected in different localities and dates (Tables 1 and 2). In Brazil, mosquitoes were collected using CDC (Centers for Disease Control) light traps with $\mathrm{CO}_{2}$ (dry ice) for $12 \mathrm{~h}$, beginning at twilight, or with Shannon trap for 3 h, from 6:00 p.m. to 9:00 p.m. Peruvian mosquitoes were collected with Shannon traps, from 6:00 p.m. to 9:00 p.m. Mosquitoes were killed with chloroform vapor and transported to the laboratory for identification, using morphological taxonomy key by Zavortink [18], Consoli and Lourenço-de-Oliveira [38], and Forattini [12]. Mosquitoes were individually stored at $-20{ }^{\circ} \mathrm{C}$ in $1.5 \mathrm{~mL}$ plastic tubes sealed with parafilm. 
Table 1. Main features of the specimens from Peru used in this study and GenBank accession numbers.

\begin{tabular}{|c|c|c|c|c|c|c|c|}
\hline $\begin{array}{l}\text { Mosquito } \\
\text { ID }\end{array}$ & Origin & $\begin{array}{l}\text { Collection } \\
\text { Year }\end{array}$ & $\begin{array}{c}\text { Species (According to } \\
\text { Morphological Identification) }\end{array}$ & $\begin{array}{l}\text { Species (According to } \\
\text { Phylogeny Analysis) }\end{array}$ & $\begin{array}{c}\mathrm{COI}{ }^{*} \\
\text { (COIF/COIR } \\
\text { Primers) }\end{array}$ & ND4 * & $\begin{array}{c}\mathrm{COI}{ }^{*} \\
\text { (LCO/HCO } \\
\text { Primers) }\end{array}$ \\
\hline $1 \mathrm{~A}$ & Miraflores, Yanatile, Calca, Cusco, Peru & 2007 & Anopheles (Kerteszia) boliviensis & Anopheles (Kerteszia) boliviensis & MH589387 & MH560305 & - \\
\hline 1B & Miraflores, Yanatile, Calca, Cusco, Peru & 2007 & Anopheles (Kerteszia) boliviensis & Anopheles (Kerteszia) boliviensis & MH589388 & MH560306 & MH589377 \\
\hline $2 \mathrm{~A}$ & Corimayo, Yanatile, Calca, Cusco, Peru & 2008 & Anopheles (Kerteszia) boliviensis & Anopheles (Kerteszia) boliviensis & MH589389 & MH560307 & - \\
\hline $2 \mathrm{~B}$ & Corimayo, Yanatile, Calca, Cusco, Peru & 2008 & Anopheles (Kerteszia) boliviensis & Anopheles (Kerteszia) boliviensis & MH589390 & MH560308 & MH589378 \\
\hline $3 \mathrm{~A}$ & Colca, Yanatile, Calca, Cusco, Peru & 2008 & Anopheles (Kerteszia) boliviensis & Anopheles (Kerteszia) boliviensis & MH589391 & MH560309 & MH589379 \\
\hline 3B & Colca, Yanatile Calca, Cusco, Peru & 2008 & Anopheles (Kerteszia) boliviensis & Anopheles (Kerteszia) boliviensis & MH589392 & MH560310 & MH589380 \\
\hline $4 \mathrm{~A}$ & Miraflores, Yanatile, Calca, Cusco, Peru & 2008 & Anopheles (Kerteszia) cruzii & Anopheles (Kerteszia) boliviensis & MH589393 & MH560311 & MH589381 \\
\hline $4 \mathrm{~B}$ & Miraflores, Yanatile, Calca, Cusco, Peru & 2008 & Anopheles (Kerteszia) cruzii & Anopheles (Kerteszia) boliviensis & MH589394 & MH563012 & MH589382 \\
\hline $5 \mathrm{~A}$ & Colca, Yanatile, Calca, Cusco, Peru & 2008 & Anopheles (Kerteszia) lepidotus & Anopheles (Kerteszia) pholidotus & MH589395 & MH563013 & - \\
\hline $5 \mathrm{~B}$ & Colca, Yanatile, Calca, Cusco, Peru & 2008 & Anopheles (Kerteszia) lepidotus & Anopheles (Kerteszia) pholidotus & MH589396 & MH560314 & - \\
\hline $6 \mathrm{~A}$ & Miraflores, Yanatile, Calca, Cusco, Peru & 2008 & Anopheles (Kerteszia) lepidotus & Anopheles (Kerteszia) boliviensis & MH589397 & MH560315 & MH589383 \\
\hline $6 \mathrm{~B}$ & Miraflores, Yanatile, Calca, Cusco, Peru & 2008 & Anopheles (Kerteszia) lepidotus & Anopheles (Kerteszia) pholidotus & MH589398 & MH563016 & MH589384 \\
\hline LAN & Selva Alegre, Alto Inambari, Sandia, Puno, Peru & 2000 & Anopheles (Kerteszia) laneanus & Anopheles (Kerteszia) boliviensis & MH589409 & - & MH589367 \\
\hline
\end{tabular}

${ }^{*} \mathrm{COI}$, cytochrome c oxidase subunit I gene; ND4, NADH dehydrogenase subunit 4 gene. 
Table 2. Main features of the specimens from Brazil used in this study and GenBank accession numbers.

\begin{tabular}{|c|c|c|c|c|c|c|c|}
\hline $\begin{array}{l}\text { Mosquito } \\
\text { ID }\end{array}$ & Origin & $\begin{array}{l}\text { Collection } \\
\text { Year }\end{array}$ & $\begin{array}{c}\text { Species (According } \\
\text { Morphological Identification) }\end{array}$ & $\begin{array}{c}\text { Species (According Phylogeny } \\
\text { Analysis) }\end{array}$ & $\begin{array}{c}\mathrm{COI} * \\
\text { (COIF/COIR } \\
\text { Primers) }\end{array}$ & ND4 * & $\begin{array}{c}\mathrm{COI}{ }^{*} \\
\text { (LCO/HCO } \\
\text { Primers) }\end{array}$ \\
\hline CA1 & Caraguatatuba, SP, Brazil & 1946 & Anopheles (Kerteszia) bellator & Anopheles (Kerteszia) cruzii & MH589406 & MH560328 & MH589361 \\
\hline B1 & Marsilac, São Paulo, SP, Brazil & 2009 & Anopheles (Kerteszia) bellator & Anopheles (Kerteszia) cruzii & - & MH560317 & MH589362 \\
\hline B2 & Marsilac, São Paulo, SP, Brazil & 2009 & Anopheles (Kerteszia) bellator & Anopheles (Kerteszia) cruzii & MH589399 & MH560318 & MH589363 \\
\hline B3 & Marsilac, São Paulo, SP, Brazil & 2009 & Anopheles (Kerteszia) bellator & Anopheles (Kerteszia) cruzii & - & MH560319 & MH589364 \\
\hline PAC1 & Parelheiros, São Paulo, SP, Brazil & 2002 & Anopheles (Kerteszia) cruzii & Anopheles (Kerteszia) cruzii & MH589412 & MH560333 & MH589352 \\
\hline PAC2 & Parelheiros, São Paulo, SP, Brazil & 2002 & Anopheles (Kerteszia) cruzii & Anopheles (Kerteszia) cruzii & MH589414 & MH560335 & MH589353 \\
\hline PAC3 & Parelheiros, São Paulo, SP, Brazil & 2002 & Anopheles (Kerteszia) cruzii & Anopheles (Kerteszia) cruzii & MH589415 & MH560336 & MH589354 \\
\hline PAC4 & Parelheiros, São Paulo, SP, Brazil & 2002 & Anopheles (Kerteszia) cruzii & Anopheles (Kerteszia) cruzii & MH589416 & MH560337 & MH589355 \\
\hline PAC5 & Parelheiros, São Paulo, SP, Brazil & 2002 & Anopheles (Kerteszia) cruzii & Anopheles (Kerteszia) cruzii & MH589417 & MH560338 & MH589356 \\
\hline PAC6 & Parelheiros, São Paulo, SP, Brazil & 2002 & Anopheles (Kerteszia) cruzii & Anopheles (Kerteszia) cruzii & MH589418 & - & MH589357 \\
\hline PAC7 & Parelheiros, São Paulo, SP, Brazil & 2002 & Anopheles (Kerteszia) cruzii & Anopheles (Kerteszia) cruzii & MH589419 & MH560339 & MH589358 \\
\hline PAC8 & Parelheiros, São Paulo, SP, Brazil & 2002 & Anopheles (Kerteszia) cruzii & Anopheles (Kerteszia) cruzii & MH589420 & MH560340 & MH589359 \\
\hline PAC9 & Parelheiros, São Paulo, SP, Brazil & 2002 & Anopheles (Kerteszia) cruzii & Anopheles (Kerteszia) cruzii & MH589421 & MH560341 & MH589360 \\
\hline PAC10X & Parelheiros, São Paulo, SP, Brazil & 2002 & Anopheles (Kerteszia) cruzii & Anopheles (Kerteszia) cruzii & MH589413 & MH560334 & - \\
\hline $\mathrm{C} 1$ & Marsilac, São Paulo, SP, Brazil & 2009 & Anopheles (Kerteszia) cruzii & Anopheles (Kerteszia) cruzii & - & MH560320 & MH589369 \\
\hline $\mathrm{C} 2$ & Marsilac, São Paulo, SP, Brazil & 2009 & Anopheles (Kerteszia) cruzii & Anopheles (Kerteszia) cruzii & MH589400 & MH560321 & MH589370 \\
\hline $\mathrm{C} 3$ & Marsilac, São Paulo, SP, Brazil & 2009 & Anopheles (Kerteszia) cruzii & Anopheles (Kerteszia) cruzii & - & MH560322 & MH589371 \\
\hline $\mathrm{C} 4$ & Marsilac, São Paulo, SP, Brazil & 2009 & Anopheles (Kerteszia) cruzii & Anopheles (Kerteszia) cruzii & MH589401 & MH560323 & MH589372 \\
\hline $\mathrm{C} 5$ & Marsilac, São Paulo, SP, Brazil & 2009 & Anopheles (Kerteszia) cruzii & Anopheles (Kerteszia) cruzii & MH589402 & MH560324 & MH589373 \\
\hline C6 & Marsilac, São Paulo, SP, Brazil & 2009 & Anopheles (Kerteszia) cruzii & Anopheles (Kerteszia) cruzii & MH589403 & MH560325 & MH589374 \\
\hline C7 & Marsilac, São Paulo, SP, Brazil & 2009 & Anopheles (Kerteszia) cruzii & Anopheles (Kerteszia) cruzii & MH589404 & MH560326 & MH589375 \\
\hline $\mathrm{C} 8$ & Marsilac, São Paulo, SP, Brazil & 2009 & Anopheles (Kerteszia) cruzii & Anopheles (Kerteszia) cruzii & MH589405 & MH560327 & MH589376 \\
\hline L1 & Campos do Jordão, SP, Brazil & 1997 & Anopheles (Kerteszia) laneanus & Anopheles (Kerteszia) laneanus & MH589407 & MH560329 & MH589365 \\
\hline L3 & Campos do Jordão, SP, Brazil & 1997 & Anopheles (Kerteszia) laneanus & Anopheles (Kerteszia) laneanus & MH589408 & MH560330 & MH589366 \\
\hline M95 & Itanhaém, SP, Brazil & 2010 & Anopheles (Kerteszia) cruzii & Anopheles (Kerteszia) cruzii & MH589411 & MH560332 & MH589386 \\
\hline M83 & Itanhaém, SP, Brazil & 2010 & $\begin{array}{c}\text { Anopheles (Anopheles) } \\
\text { maculipes/pseudomaculipes }\end{array}$ & Anopheles (Anopheles) intermedius & MH589410 & MH560331 & MH589385 \\
\hline PAN1 & Parelheiros, São Paulo, SP, Brazil & 2002 & Anopheles (Nyssorhynchus) sp. & Anopheles (Nysssorhynchus) strodei & - & MH560342 & MH589368 \\
\hline
\end{tabular}

* COI, cytochrome c oxidase subunit I gene; ND4, NADH dehydrogenase subunit 4 gene. 


\subsection{Genomic DNA Extraction and PCR Amplification of Mitochondrial Gene Fragments}

Mosquitoes were crushed with disposable tips in lysis buffer, and genomic DNA (gDNA) was obtained using PureLink Genomic DNA Purification Kit (Invitrogen), according to the manufacturer's instructions.

A fragment of 710 base pairs (bp) of the barcode region of the mitochondrial COI gene was generated by PCR amplification using the primer pair LCO1490 and HCO2198 [39], following the protocol proposed by Ruiz et al. [40]. A fragment of $\approx 600 \mathrm{bp}$ of COI gene (gene region used in phylogeny) was obtained as described [41] with the primers COIF (5'-GGA TTA TTA GGA TTT ATT GT- $\left.3^{\prime}\right)$ and COIR ( $5^{\prime}$-GCA AAT AAT GAA ATT GTT CT- $\left.3^{\prime}\right)$. A fragment of $\approx 400 \mathrm{bp}$ of the mitochondrial ND4 gene was generated using the primers ND4+ (5'-GTD YAT TTA TGA TTR CCT AA-3') and ND4-(5'-CTT CGD CTT CCW ADW CGT TC-3') and the PCR program, as described [42].

\subsection{Sequencing, Alignment, and Phylogenetic Analysis}

Amplified fragments were sequenced directly in both directions by BigDye Terminator v3.0 Cycle Sequencing Kit in ABI Genetic Analyzer (Applied Biosystems ${ }^{\circledR}$, Foster City, CA, USA), using the corresponding flanking primers. The COI and ND4 sequences obtained from newly sequenced mosquitoes were deposited in the GenBank database (COI: MH589352-MH589421; ND4: MH560305-MH560342). Sequences were aligned with the reference sequences (Table 3) using Clustal X version 1.81 [43]. Alignments were then inspected and edited in MEGA version 5.1 [44].

Table 3. GenBank accession numbers of the reference mosquitoes used in the study.

\begin{tabular}{|c|c|c|c|}
\hline Species & Mosquito ID & Origin & \# GenBank \\
\hline An. lepidotus & 51917879 & Napo/Ecuador & JQ041282* \\
\hline An. lepidotus & 51917894 & Napo/Ecuador & JQ041284* \\
\hline An. pholidotus & 51917595 & Tachira/Venezuela & JQ041287 * \\
\hline An. pholidotus & 51917607 & Tachira/Venezuela & JQ041288* \\
\hline An. homunculus & BA22_33 & Camacan, BA/Brazil & JQ291234 * \\
\hline An. homunculus & ES10_2 & Santa Teresa, ES/Brazil & JQ291235 * \\
\hline An. homunculus & RS20_21 & Maquiné, RS/Brazil & JQ291236 * \\
\hline An. homunculus & RS20_33 & Maquiné, RS/Brazil & JQ291237* \\
\hline An. homunculus & RS30_56 & Maquiné, RS/Brazil & JQ291238* \\
\hline An. homunculus & SP23_1 & Cananéia, SP/Brazil & JQ291240* \\
\hline An. homunculus & ST26 & Cananéia, SP/Brazil & JQ291242 * \\
\hline An. lepidotus & CCDB-10764-A9 & Orellana/Ecuador & KF670991 * \\
\hline An. cruzii & ??? & Cananéia/SP & KJ701506 \\
\hline An. cruzii & 104 & Tapiraí, SP/Brazil & KM507293 * \\
\hline An. cruzii & 110 & Tapiraí, SP/Brazil & KM507294* \\
\hline An. cruzii & 112 & Tapiraí, SP/Brazil & KM507295* \\
\hline An. cruzii & 127 & Tapiraí, SP/Brazil & KM507296* \\
\hline An. cruzii & 192 & Tapiraí, SP/Brazil & KM507297* \\
\hline An. cruzii & 198 & Tapiraí, SP/Brazil & KM507298* \\
\hline An. cruzii & 199 & Tapiraí, SP/Brazil & KM507299* \\
\hline An. cruzii & 200 & Tapiraí, SP/Brazil & KM507300* \\
\hline An. cruzii & 217 & Tapiraí, SP/Brazil & KM507301* \\
\hline An. cruzii & 240 & Tapiraí, SP/Brazil & KM507302 * \\
\hline An. cruzii & 241 & Tapiraí, SP/Brazil & KM507303 * \\
\hline An. cruzii & 242 & Tapiraí, SP/Brazil & KM507304 * \\
\hline An. cruzii & 255 & Tapiraí, SP/Brazil & KM507305 * \\
\hline An. cruzii & 257 & Tapiraí, SP/Brazil & KM507306 * \\
\hline An. cruzii & 258 & Tapiraí, SP/Brazil & KM507307* \\
\hline An. cruzii & 259 & Tapiraí, SP/Brazil & KM507308 * \\
\hline An. cruzii & 260 & Tapiraí, SP/Brazil & KM507309* \\
\hline
\end{tabular}


Table 3. Cont.

\begin{tabular}{cccc}
\hline Species & Mosquito ID & Origin & \# GenBank \\
\hline An. cruzii & 261 & Tapiraí, SP/Brazil & KM507310* \\
An. cruzii & 262 & Tapiraí, SP/Brazil & KM507311* \\
An. cruzii & 267 & Tapiraí, SP/Brazil & KM507312* \\
An. cruzii & 269 & Tapiraí, SP/Brazil & KM507314* \\
An. homunculus & BA22_32 & Camacan/BA & KU551283 \\
An. cruzii & PEC___7 & São Paulo/SP & KU551284 \\
An. cruzii & RJ03_2 & Itatiaia/RJ & KU551285 \\
An. cruzii & RS32_11_7 & Maquiné/RS & KU551286 \\
An. bellator & SP24_3_1 & Cananéia/SP & KU551287 \\
An. laneanus & SP52-103 & Pindamonhangaba/SP & KU551288 \\
An. cruzii & ST16 & Cananéia/SP & KU551289 \\
An. neivai & MB10165 & Petit-Saut/French Guyana & MF172273* \\
An. neivai & MB10252 & Petit-Saut/French Guyana & MF172274* \\
An. neivai & MB10253 & Petit-Saut/French Guyana & MF172275* \\
An. neivai & MB10254 & Petit-Saut/French Guyana & MF172276* \\
An. homunculus & BA22_31 & Camacan/BA & MF381605 \\
An. laneanus & SP52-17 & Pindamonhangaba/SP & MF381613 \\
An. homunculus & RS30_158 & Maquiné/RS & MF381697 \\
An. (An.) intermedius & SP02_8_1 & Pariquera-Açú/SP & MF381700 \\
An. (Nyr.) strodei & SP104_18_1 & Pindamonhangaba/SP & MF381724* \\
An. homunculus & ST19 & Cananéia/SP & MF381729 \\
\hline
\end{tabular}

* Only for COI barcoding region analysis. \# GenBank accession number (https://www.ncbi.nlm.nih.gov/genbank/).

Two methods of phylogenetic reconstruction were implemented using the Bayesian approach. In the first round of analysis, $624 \mathrm{bp}$ of the COI barcoding region was employed, due the higher availability of sequences of this COI gene region for the Anopheles diversity in the GenBank database, from which all the sequence data of reference Kerteszia species were downloaded. In the second round of analysis, phylogenetic relationship was investigated using ND4 and COI genes. COI and ND4 datasets were concatenated, after trimming the primers off from the sequences-including $338 \mathrm{bp}$ of the ND4 gene, $511 \mathrm{bp}$ of the COI, and $624 \mathrm{bp}$ of the barcode region of the COI, generating a total of $1473 \mathrm{bp}$. In this analysis only reference sequences from complete mtDNA were used, avoiding an incomplete final matrix and missing data. All sequence accession numbers used in both analyses are provided in Tables 1-3 and added into phylogenetic trees.

The phylogenetic reconstructions were performed using the Bayesian approach implemented in MrBayes v3.2.0 [45]. Bayesian inferences were run with two Markov Chain Monte Carlo searches of 3 million generations, each with sampling of 1 in 300 trees. After a burn-in of $25 \%$, the remaining 15,002 trees were used to generate a 50\% majority-rule consensus tree. The results of analyses were visualized using FigTree version 1.4.0 [46]. The topologies were rooted using sequences obtained for species of the subgenera Anopheles or Nyssorhynchus (Tables 2 and 3).

\section{Results}

The results of the analyses using only DNA sequences of the COI barcoding region are shown in Figure 1. This approach was used with the aim of increasing the number of sequences in the phylogenetic tree, as there are no complete mitochondrial sequences available for some Kerteszia species such as An. neivai, An. pholidotus, and An. lepidotus. The sequence alignment composed of 85 sequences with 624 bp length -9 from Peru, 26 from Brazil, and 50 references from GenBank-had 168 variable sites. The Bayesian phylogenetic tree (Figure 1) shows that the sequences from nine specimens from Peru grouped together in a strongly supported clade. The Peruvian species clade contains two major groups. One group encompasses sequences from specimens identified as An. boliviensis, An. cruzii, An. laneanus, and An. lepidotus. The second group contains sequences from An. lepidotus that clustered with An. pholidotus from Venezuela. 


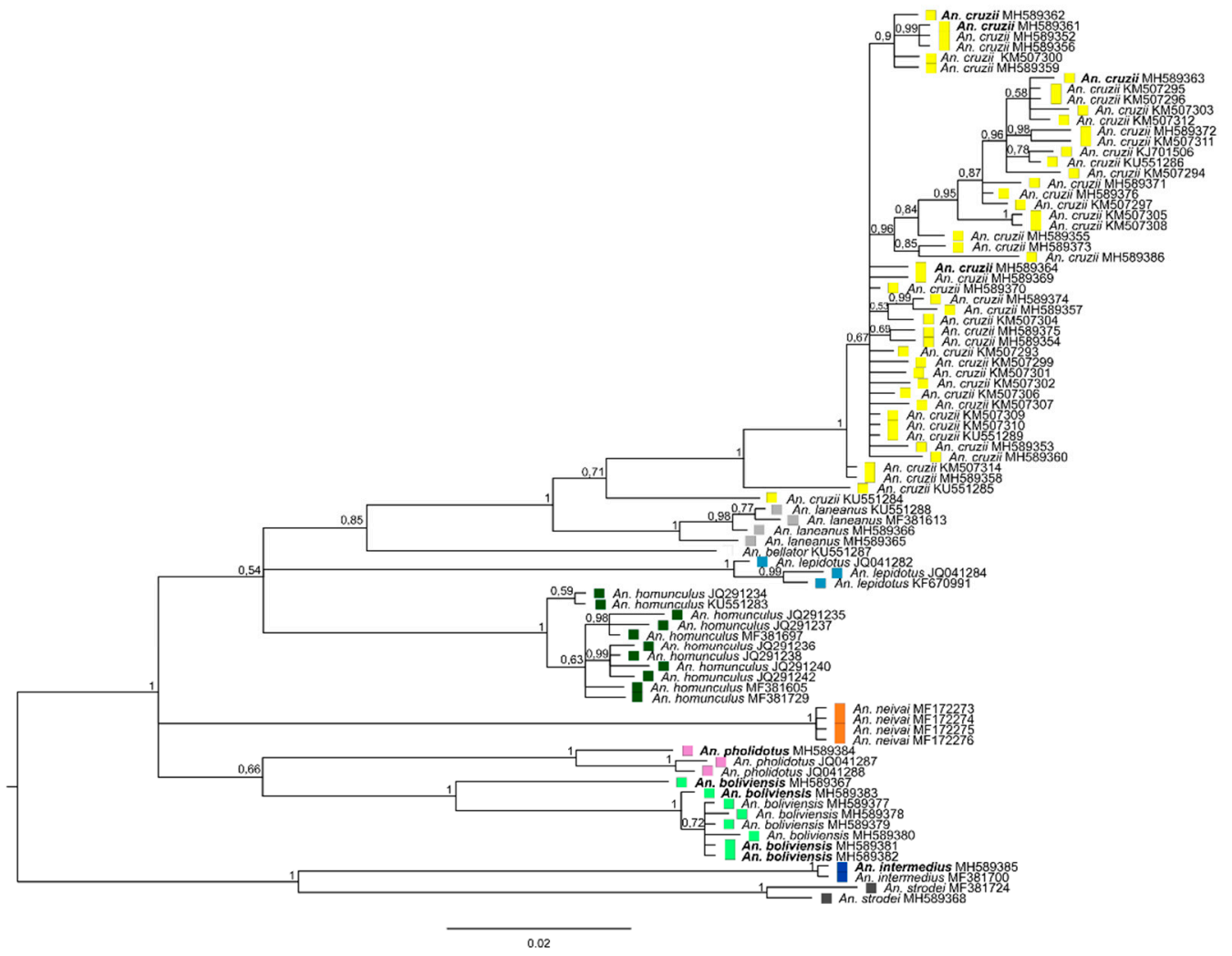

Figure 1. Phylogeny of the Kerteszia species using cytochrome c oxidase I (COI) barcoding region by Bayesian inference tree. Posterior probability values are shown to each clade. Yellow, An. cruzii clade; grey, An. laneanus clade; white, An. bellator; blue, An. lepidotus; dark green, An. homunculus; orange, An. neivai; pink, An. pholidotus; green, An. boliviensis; dark blue, An. (Anopheles) species; dark grey, An. (Nyssorhynchus) species. Specimens reclassified are highlighted in bold (see Tables 1 and 2).

For the second round of Bayesian analysis, approximately $1.5 \mathrm{~kb}(10 \%$ of the whole mtDNA) was employed, including COI and ND4 genes of 39 specimens of An. (Kerteszia) species from Peru and Brazil. Twelve new sequences were blasted with sequences available in GenBank for other Kerteszia species to verify identification and potential presence of stop codon in each sequence. The alignment generated $1473 \mathrm{bp}$ with 371 variable sites. Sequences of Anopheles (Anopheles) species were used as outgroup. All clades recovered in the Bayesian phylogenetic analysis were strongly supported (Bayesian Posterior Probability-BPP =1). All the Anopheles (Kerteszia) species were grouped in a clade that was separated into two clades: one containing all Peruvian species, and the second clade being formed of species collected in Brazil (Figure 2). The Peruvian species clade was split in two minor groups. Species from Brazil were separated in two clades: one clade composed of An. bellator and An. homunculus, and a second clade composed of An. laneanus and An. cruzii, with four mosquitoes morphologically identified as An. bellator (one of which was collected in 1946). Sequences of An. cruzii from Brazil were separated into clades in accordance with the geographical localities of Serra do Mar, Serra da Mantiqueira and Serra da Cantareira (Figures 2 and 3). 


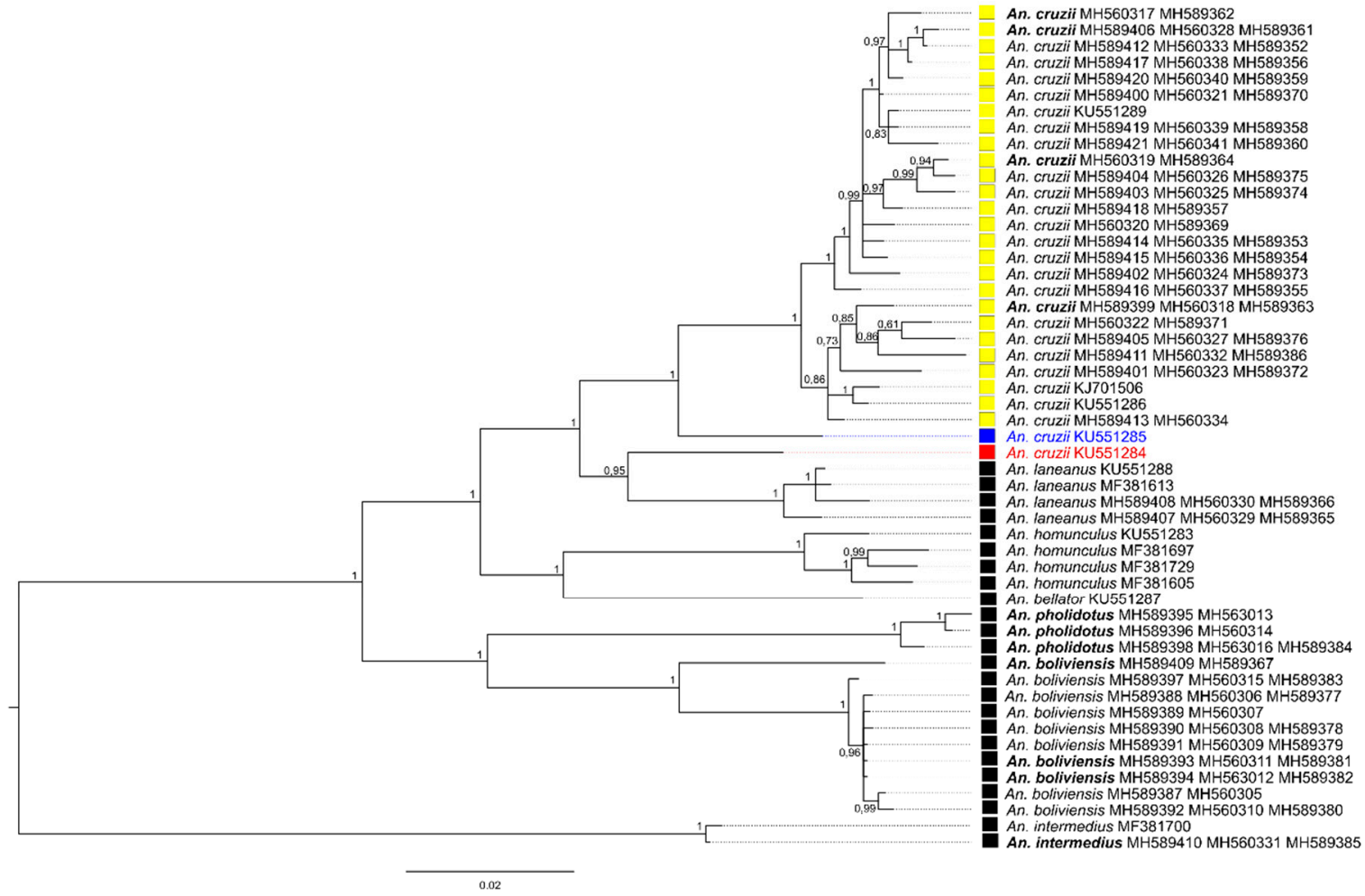

Figure 2. Phylogeny of the Kerteszia species using COI and NADH dehydrogenase subunit 4 (ND4) genes by Bayesian inference tree. Posterior probability values are shown to each clade. An. cruzii from is Serra da Cantareira represented in red. An. cruzii from Serra da Mantiqueira is represented in blue. An. cruzii from Serra do Mar is represented as a yellow box. Specimens reclassified are highlighted in bold (see Tables 1 and 2).

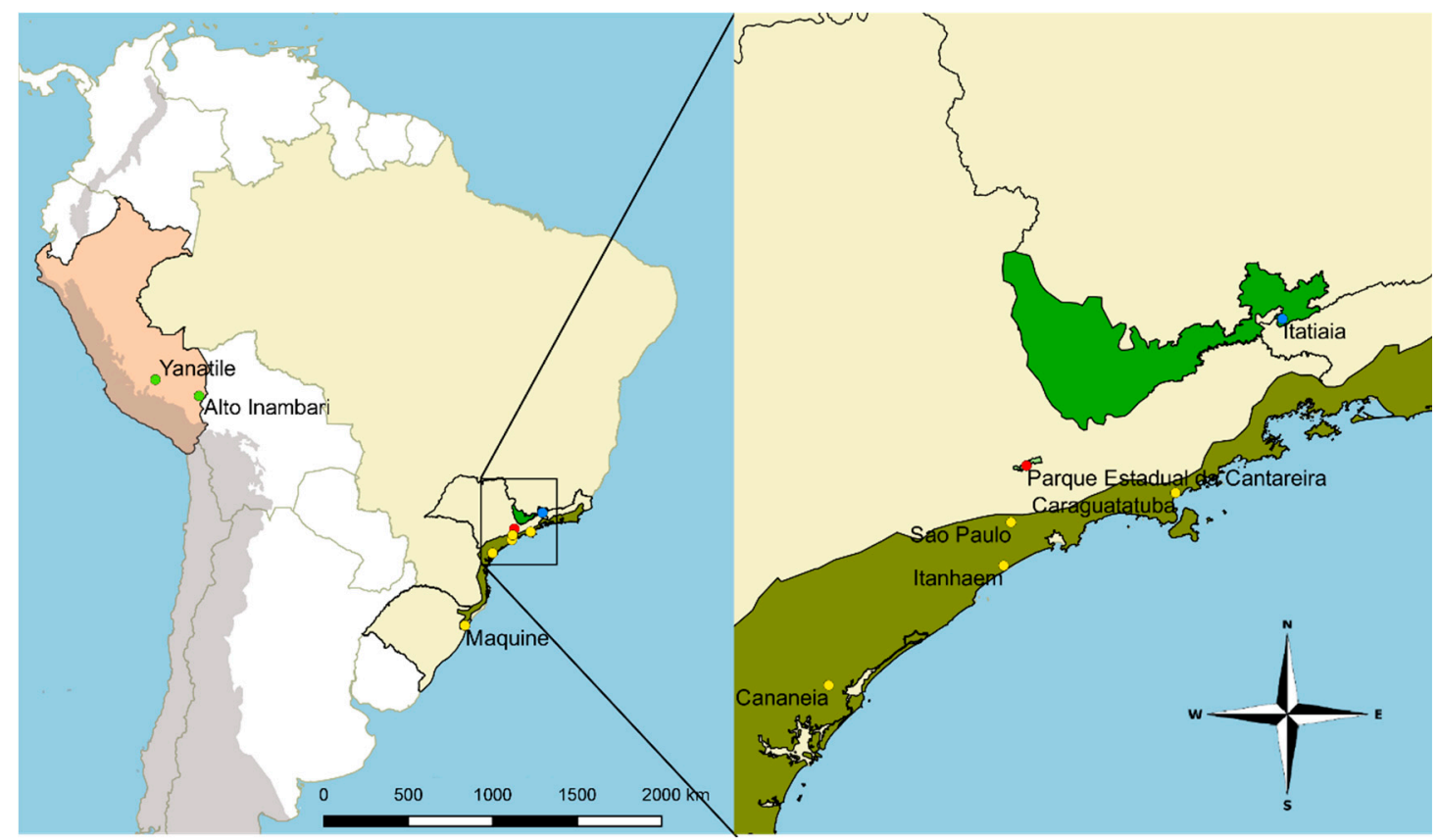

Figure 3. (a) Origin localities map for An. cruzii sequences from Peru and Brazil used in this study. (b) Localities of Itatiaia/RJ (Serra da Mantiqueira, blue dot); Parque Estadual da Cantareira/SP (Serra da Cantareira, red dot); Cananéia/SP, Caraguatatuba/SP, Itanhaém/SP, and São Paulo/SP (all in Serra do Mar, yellow dots) are shown. 


\section{Discussion}

Recently, the number of publications using mitochondrial sequences for inferring phylogenetic relationships within the Kerteszia has increased [47-53]. However, there are few studies focusing on populations of An. cruzii $[19,27,54]$, with no publication regarding Kerteszia species that occur in Peru. In order to provide relevant information about diversity and improve knowledge in taxonomy of Kerteszia species from Peru, we sequenced two mitochondrial genes (COI and ND4), including the DNA barcode region.

The analysis of genetic differentiation of COI and ND4 genes indicated that there are at least two Kerteszia species of that occur in Peru. Preliminary identification of specimens of An. cruzii and An. lepidotus using morphological characters of the female was not confirmed by phylogenetic analyses of mitochondrial genes. In fact, specimens from Peru that were identified as An. cruzii had scales on the abdominal terga, indicating that they belonged to other species. In the Bayesian topology, those specimens of An. cruzii with scales on the abdomen terga grouped with most of the mosquitoes from Peru, which were identified as An. boliviensis. In addition, one specimen morphologically identified as An. lepidotus clustered with An. pholidotus, with an identity of $98 \%$ in the COI barcoding region. The clade encompassing $A n$. pholidotus sequences was recovered and was well separated from An. lepidotus. Moreover, a Peruvian specimen preliminary identified as An. laneanus clustered with An. boliviensis. For accurate identification of An. boliviensis, An. pholidotus, and An. lepidotus using morphology, it is important to examine characters of the male and fourth-instar larva in addition to female characters [18]. Furthermore, until 2012 [25], the only morphological feature that had been proposed to differentiate females of An. pholidotus from An. lepidotus was the size of the scales on proximal tergites, being moderately wide or broad for An. lepidotus and predominantly narrow to moderately wide for An. pholidotus [18]. This characteristic was shown vague and difficult to interpret. In fact, in other countries of South America also, such as Colombia, molecular analyses showed that the species previously believed to be An. lepidotus corresponded to An. pholidotus [47].

Chromosomal banding pattern of the ovarian polytene chromosomes had already suggested that there are three putative species under the name An. cruzii, termed as A, B, and C [26,55]. More recently, studies employing DNA sequences from nuclear genes have corroborated this hypothesis [56-59]. Our analysis, using DNA sequences from mitochondrial genes, also supported the hypotheses of An. cruzii representing a complex of morphologically similar species. The sequences employed in this study that were generated from specimens identified as An. cruzii were separated in accordance to their geographical origin. Sequence with the GenBank code KU551284 was recovered in a clade that is sister to An. laneanus and yet separate from other specimens of An. cruzii. This KU551284 sequence was obtained from a mosquito collected in the Parque da Serra da Cantareira, situated in the mountain range north of the municipality of São Paulo [27]. The KU551285 sequence was also sorted separately from all the other cruzii sequences and was obtained from a mosquito collected in Itatiaia National Park, in the Mantiqueira mountain range, between the states of Rio de Janeiro and Minas Gerais [27]. All the other An. cruzii sequences were grouped in a branch composed by sequences from mosquitoes collected only in the Serra do Mar region, a mountainous chain that extends by approximately $1500 \mathrm{~km}$ along the coast, from Rio de Janeiro state to the north of Santa Catarina state. Indeed, at least two genetically distinct An. cruzii lineages have been shown to occur in the mountains covered by the Atlantic Forest in South-East Brazil [60]. Here, through another methodology, we confirmed these two populations and showed that a third, coming from another mountain range, the Serra da Cantareira, also separates from the other two in the phylogenetic trees. These three populations of An. cruzii were consistently separated regardless of the methodology used (analysis of mitochondrial or nuclear genes). Moreover, in our phylogenetic trees, the addition of another COI gene region as well as another mitochondrial gene (ND4) has not changed the topology of the tree, showing that the most important information is contained in the barcoding region, confirming that the COI gene is more useful for identifying sibling and cryptic species, as has been demonstrated [51]. 
We also demonstrated that mosquitoes morphologically identified as An. bellator (CA1, B1, B2, and B3) are likely An. cruzii. In fact, adult females of these species are morphologically similar and identification can be problematic when the adults were damaged during field collections [18]. In addition to the placement of these mosquitoes on the phylogenetic trees, their grouping based on the geographical region of the field collections confirms that An. cruzii is a species complex.

\section{Conclusions}

The analysis of genetic differentiation of COI and ND4 genes indicated that there are at least two Kerteszia species occurring in Peru: An. boliviensis and An. pholidotus. The occurrence of these species in Peru is herein registered for the first-time, using COI DNA sequences. Moreover, we confirmed the occurrence of two subpopulations of An. cruzii in the Atlantic rainforest and showed a third population in the mountain range of the Parque da Serra da Cantareira. This population is likely a distinct species that clustered separately from the remaining two lineages of An. cruzii in the phylogenetic trees. One lineage encompasses specimens from Serra do Mar on the Atlantic coast, and the second lineage is formed by An. cruzii from Serra da Mantiqueira. Besides their placement in the phylogenetic tree, the grouping of An. cruzii sequences reflects the geographical origin of the adults (Serra da Cantareira, Serra do Mar, and Serra da Mantiqueira). Thus, these findings add additional support for the An. cruzii species complex.

Author Contributions: Conceptualization, K.K.; formal analysis, K.K. and M.A.M.S.; investigation, L.d.O.G.; resources, K.K., H.H.Y.T., F.R.A., A.G.C., A.M.R.d.C.D., R.d.S.M., and R.M.T.; writing-original draft, K.K. and L.d.O.G.; writing-review and editing, K.K. and M.A.M.S. All authors have read and agreed to the published version of the manuscript.

Funding: We thank the Vicerrectorado for Research of the Universidad Nacional Mayor de San Marcos for their sponsorship (research code: 100101171). M.A.M.S. is financially supported by Fundação de Amparo à Pesquisa do Estado de São Paulo (FAPESP), grant number 2014/26229-7, and Conselho Nacional de Pesquisa (CNPq), grant number 301877/2016-5. A.M.R.d.C.D. is supported by grant FAPESP number 2008/52016-0. L.O.G. is supported by a postdoctoral fellowship (FAPESP 2018/16232-1). K.K. is a CNPq research fellow, grant number 308678/2018-4.

Acknowledgments: We thank Bruno Mathias for his help in editing the figures.

Conflicts of Interest: The authors declare no conflict of interest.

\section{References}

1. World Health Organization. World Malaria Report; WHO: Geneva, Switzerland, 2016.

2. Centro Nacional de Epidemiología, Prevención y Control de Enfermedades-MINSA, Perú. Available online: http://www.dge.gob.pe/portal/docs/vigilancia/sala/2017/SE51/malaria.pdf (accessed on 22 May 2019).

3. Pan American Health Organization; World Health Organization. Epidemiological Update: Malaria in the Americas; PAHO: Washington, DC, USA; WHO: Geneva, Switzerland, 2019.

4. Recht, J.; Siqueira, A.M.; Monteiro, W.M.; Herrera, S.M.; Herrera, S.; Lacerda, M.V.G. Malaria in Brazil, Colombia, Peru and Venezuela: Current challenges in malaria control and elimination. Malar. J. 2017, 16, 273. [CrossRef]

5. Sulzer, A.J.; Cantella, R.; Colichon, A.; Gleason, N.N.; Walls, K.W. A focus of hyperendemic Plasmodium malariae-P. vivax with no P. falciparum in a primitive population in the Peruvian Amazon jungle. Bull. World Health Organ. 1975, 52, 273-278.

6. Aramburu, G.J.; Asayag, C.R.; Witzig, R. Malaria reemergence in the peruvian amazon region. Emerg. Infect. Dis. 1999, 5, 209-215.

7. Manguin, S.R.; Wilkerson, R.C.; Conn, J.E.; Rubio-Palis, Y.; Danoff-Burg, J.A.; Roberts, D.R. Population structure of the primary malaria vector in South America, Anopheles darlingi, using isoenzyme, ITS2, RAPD, and morphological markers. Am. J. Trop. Med. Hyg. 1999, 60, 364-376. [CrossRef] [PubMed]

8. Flores, C.; Fernández, R.; Escobedo, K.S.; Vela, Q.; Schoeler, G.B. Natural Plasmodium infections in Anopheles darlingi and Anopheles benarrochi (Diptera: Culicidae) from Eastern Peru. J. Med. Entomol. 2004, 41, 489-494. [CrossRef] [PubMed] 
9. Reinbold-Wasson, D.D.; Sardelis, M.R.; Jones, J.W.; Watts, D.M.; Fernandez, R.; Carbajal, F.; Pecor, J.E.; Calampa, C.; Klein, T.A.; Turell, M.J. Determinants of Anopheles seasonal distribution patterns across a forest to periurban gradient near Iquitos, Peru. Am. J. Trop. Med. Hyg. 2012, 86, 459-463. [CrossRef] [PubMed]

10. Moreno, M.; Saavedra, M.P.; Bickersmith, S.A.; Prussing, C.; Michalski, A.; Tong Rios, C.; Vinetz, J.M.; Conn, J.E. Intensive trapping of blood-fed Anopheles darlingi in Amazonian Peru reveals unexpectedly high proportions of avian blood-meals. PLoS Negl. Trop. Dis. 2017, 11, e0005337. [CrossRef] [PubMed]

11. Oliveira-Ferreira, J.; Lacerda, M.V.; Brasil, P.; Ladislau, J.L.; Tauil, P.L.; Daniel-Ribeiro, C.T. Malaria in Brazil: An overview. Malar. J. 2010, 9, 115. [CrossRef]

12. Forattini, O. P Culicidologia Médica, vol. 2: Identificação, Biologia, Epidemiologia; Edusp: São Paulo, Brazil, 2002.

13. Carlos, B.C.; Rona, L.D.P.; Christophides, G.K.; Souza-Neto, J.A. A comprehensive analysis of malaria transmission in Brazil. Pathog. Glob. Health 2019, 113, 1-13. [CrossRef]

14. Deane, L.M.; Ferreira-Neto, J.A.; Deane, S.P.; Silveira, I.P. Anopheles (Kerteszia) cruzii, a natural vector of the monkey malaria parasites, Plasmodium simium and Plasmodium Brasilianum. Trans. R. Soc. Trop. Med. Hyg. 1970, 64, 647. [CrossRef]

15. Deane, L.M.; Ferreira-Neto, J.A.; Lima, M.M. The vertical dispersion of Anopheles (Kerteszia) cruzii in a forest in southern Brazil suggests that human cases of malaria of simian origin might be expected. Mem. Inst. Oswaldo Cruz 1984, 79, 461-463. [CrossRef] [PubMed]

16. Demari-Silva, B.; Laporta, G.Z.; Oliveira, T.; Sallum, M. Plasmodium infection in Kerteszia cruzii (Diptera: Culicidae) in the Atlantic tropical rain forest, southeastern Brazil. Infect. Genet. Evol. 2020, 78, 104061. [CrossRef] [PubMed]

17. Multini, L.C.; Wilke, A.B.B.; Marrelli, M.T. Neotropical Anopheles (Kerteszia) mosquitoes associated with bromeliad-malaria transmission in a changing world. Acta Trop. 2020, 205, 105413. [CrossRef] [PubMed]

18. Zavortink, T.J. Mosquito studies XXIX. A review of the subgenus Kerteszia of Anopheles. Contr. Amer. Entomol. Inst. 1973, 9, 1-54.

19. Foster, P.G.; de Oliveira, T.M.P.; Bergo, E.S.; Conn, J.E.; Sant'Ana, D.C.; Nagaki, S.S.; Nihei, S.; Lamas, C.E.; González, C.; Moreira, C.C.; et al. Phylogeny of Anophelinae using mitochondrial protein coding genes. R. Soc. Open Sci. 2017, 4, 170758. [CrossRef] [PubMed]

20. Collucci, E.; Sallum, M.A.M. Phylogenetic analysis of the subgenus Kerteszia of Anopheles (Diptera: Culicidae: Anophelinae) based on morphological characters. Insect Syst. Evol. 2003, 34, 361-372. [CrossRef]

21. Marrelli, M.T.; Malafronte, R.S.; Sallum, M.A.; Natal, D. Kerteszia subgenus of Anopheles associated with the Brazilian Atlantic rainforest: Current knowledge and future challenges. Malar. J. 2007, 6, 127. [CrossRef]

22. Walter Reed Biosystematics Unit (WRBU). Systematic Catalog of Culicidae. 2019. Available online: http://www.mosquitocatalog.org/taxon_descr.aspx?ID=16082 (accessed on 31 August 2019).

23. Guimarães, J.H. Systematic Database of Diptera of the Americas South of the United States (Family Culicidae); Plêiade/FAPESP: São Paulo, Brazil, 1997.

24. Sallum, M.A.M.; Dos Santos, C.L.S.; Wilkerson, R.C. Studies on Anopheles (Kerteszia) homunculus Komp (Diptera: Culicidae). Zootaxa 2009, 2299, 1-18. [CrossRef]

25. Harrison, B.A.; Ruiz-Lopez, F.; Falero, G.C.; Savage, H.M.; Pecor, J.E.; Wilkerson, R.C. Anopheles (Kerteszia) lepidotus (Diptera: Culicidae), not the malaria vector we thought it was: Revised male and female morphology; larva, pupa, and male genitalia characters; and molecular verification. Zootaxa 2012, 3218, 1-17. [CrossRef]

26. Ramirez, C.C.; Dessen, E.M. Chromosome differentiated populations of Anopheles cruzii: Evidence for a third sibling species. Genetica 2000, 108, 73-80. [CrossRef]

27. Oliveira, T.M.; Foster, P.G.; Bergo, E.S.; Nagaki, S.S.; Sanabani, S.S.; Marinotti, O.; Marinotti, P.N.; Sallum, M.A. Mitochondrial Genomes of Anopheles (Kerteszia) (Diptera: Culicidae) From the Atlantic Forest, Brazil. J. Med. Entomol. 2016, 53, 790-797. [CrossRef] [PubMed]

28. Multini, L.C.; Marrelli, M.T.; Beier, J.C.; Wilke, A.B.B. Increasing Complexity Threatens the Elimination of Extra-Amazonian Malaria in Brazil. Trends Parasitol. 2019, 35, 383-387. [CrossRef] [PubMed]

29. Ruiz-Lopez, F.; Wilkerson, R.C.; Conn, J.E.; McKeon, S.N.; Levin, D.M.; Quiñones, M.L.; Póvoa, M.M.; Linton, Y.M. DNA barcoding reveals both known and novel taxa in the albitarsis group (Anopheles: Nyssorhynchus) of Neotropical malaria vectors. Parasit. Vectors 2012, 5, 44. [CrossRef] [PubMed]

30. Bourke, B.P.; Oliveira, T.P.; Suesdek, L.; Bergo, E.S.; Sallum, M.A. A multi-locus approach to barcoding in the Anopheles strodei subgroup (Diptera: Culicidae). Parasit. Vectors 2013, 6, 111. [CrossRef] [PubMed] 
31. Hebert, P.D.N.; Cywinska, A.; Ball, S.L.; deWaard, J.R. Biological identifications through DNA barcodes. Proc. Biol. Sci. 2003, 270, 313-321. [CrossRef]

32. Hebert, P.D.N.; Ratnasingham, S.; deWaard, J.R. Barcoding animal life: Cytochrome c oxidase subunit 1 divergences among closely related species. Proc. Biol. Sci. 2003, 270, S96-S99. [CrossRef]

33. Cywinska, A.; Hunter, F.F.; Hebert, P.D.N. Identifying Canadian mosquito species through DNA barcodes. Med. Vet. Entomol. 2006, 20, 413-424. [CrossRef]

34. Kumar, N.P.; Rajavel, A.R.; Natarajan, R.; Jambulingam, P. DNA barcodes can distinguish species of Indian mosquitoes (Diptera: Culicidae). J. Med. Entomol. 2007, 44, 1-7. [CrossRef]

35. Mirabello, L.; Conn, J.E. Molecular population genetics of the malaria vector Anopheles darlingi in Central and South America. Heredity 2006, 96, 311-321. [CrossRef]

36. Gutiérrez, L.; Orrego, L.M.; Gómez, G.F.; López, A.; Luckhart, S.; Conn, J.E.; Correa, M.M. A new mtDNA COI gene lineage closely related to Anopheles janconnae of the Albitarsis complex in the Caribbean region of Colombia. Mem. Inst. Oswaldo Cruz 2010, 105, 1019-1025. [CrossRef]

37. Jaramillo, L.M.; Gutiérrez, L.A.; Luckhart, S.; Conn, J.E.; Correa, M.M. Molecular evidence for a single taxon, Anopheles nuneztovari s.l., from two endemic malaria regions in Colombia. Mem. Inst. Oswaldo Cruz 2011, 106, 1017-1023. [CrossRef] [PubMed]

38. Consoli, R.A.G.B.; Lourenço-de-Oliveira, R. Principais Mosquitos de Importância Sanitária No Brasil; Editora Fiocruz: Rio de Janeiro, Brazil, 1994.

39. Folmer, O.; Black, M.; Hoeh, W.; Lutz, R.; Vrijenhoek, R. DNA primers for amplification of mitochondrial cytochrome c oxidase subunit I from diverse metazoan invertebrates. Mol. Mar. Biol. Biotechnol. 1994, 3 , 294-299. [PubMed]

40. Ruiz, F.; Linton, Y.M.; Ponsonby, D.J.; Conn, J.E.; Herrera, M.; Quiñones, M.L.; Vélez, I.D.; Wilkerson, R.C. Molecular comparison of topotypic specimens confirms Anopheles (Nyssorhynchus) dunhami Causey (Diptera: Culicidae) in the Colombian Amazon. Mem. Inst. Oswaldo Cruz 2010, 105, 899-903. [CrossRef] [PubMed]

41. Sallum, M.A.M.; Schultz, T.R.; Foster, P.G.; Aronstein, K.; Wirtz, R.A.; Wilkerson, R.C. Phylogeny of Anophelinae (Diptera: Culicidae) based on nuclear ribosomal and mitochondrial DNA sequences. Syst. Entomol. 2002, 27, 361-382. [CrossRef]

42. Gorrochotegui-Escalante, N.; Munoz, M.L.; Fernandez-Salas, I.; Beaty, B.J.; Black, W.C. 4th. Genetic isolation by distance among Aedes aegypti populations along the northeastern coast of Mexico. Am. J. Trop. Med. Hyg. 2000, 62, 200-209. [CrossRef] [PubMed]

43. Thompson, J.D.; Gibson, T.J.; Plewniak, F.; Jeanmougin, F.; Higgins, D.G. The CLUSTAL_X windows interface: Flexible strategies for multiple sequence alignment aided by quality analysis tools. Nucleic Acids Res. 1997, 25, 4876-4882. [CrossRef]

44. Tamura, K.; Peterson, D.; Peterson, N.; Stecher, G.; Nei, M.; Kumar, S. MEGA5: Molecular Evolutionary Genetics Analysis Using Maximum Likelihood, Evolutionary Distance, and Maximum Parsimony Methods. Mol. Biol. Evol. 2011, 28, 2731-2739. [CrossRef]

45. Huelsenbeck, J.P.; Ronquist, F. MRBAYES: Bayesian inference of phylogeny. Bioinformatics 2001, 17, 754-755. [CrossRef]

46. Rambaut, A. FigTree: Tree Figure Drawing Tool Version 1.4.0, Institute of Evolutionary Biology, University of Edinburgh. 2006-2012. Available online: http://tree.bio.ed.ac.uk/software/figtree (accessed on 22 May 2019).

47. Escovar, J.E.; González, R.; Quiñones, M.L.; Wilkerson, R.C.; Ruiz, F.; Harrison, B.A. Morphology of the larvae, male genitalia and DNA sequences of Anopheles (Kerteszia) pholidotus (Diptera: Culicidae) from Colombia. Mem. Inst. Oswaldo Cruz 2014, 109, 473-479. [CrossRef]

48. Freitas, L.A.; Russo, C.A.; Voloch, C.M.; Mutaquiha, O.C.; Marques, L.P.; Schrago, C.G. Diversification of the Genus Anopheles and a Neotropical Clade from the Late Cretaceous. PLoS ONE 2015, 10, e0134462. [CrossRef]

49. Hua, Y.Q.; Ding, Y.R.; Yan, Z.T.; Si, F.L.; Luo, Q.C.; Chen, B. The complete mitochondrial genome of Anopheles minimus (Diptera: Culicidae) and the phylogenetics of known Anopheles mitogenomes. Insect Sci. 2016, 23, 353-365. [CrossRef] [PubMed]

50. López-Rubio, A.; Suaza-Vasco, J.; Marcet, P.L.; Ruíz-Molina, N.; Cáceres, L.; Porter, C.; Uribe, S. Use of DNA barcoding to distinguish the malaria vector Anopheles neivai in Colombia. Zootaxa 2016, 4175, 377-389. [CrossRef] [PubMed] 
51. Wang, G.; Li, C.; Zheng, W.; Song, F.; Guo, X.; Wu, Z.; Luo, P.; Yang, Y.; He, L.; Zhao, T. An evaluation of the suitability of COI and COII gene variation for reconstructing the phylogeny of, and identifying cryptic species in, anopheline mosquitoes (Diptera Culicidae). Mitochondrial Dna A Dna Mapp. Seq. Anal. 2017, 28, 769-777. [CrossRef] [PubMed]

52. Chen, K.; Wang, Y.; Li, X.Y.; Peng, H.; Ma, Y.J. Sequencing and analysis of the complete mitochondrial genome in Anopheles sinensis (Diptera: Culicidae). Infect. Dis. Poverty 2017, 6, 149. [CrossRef] [PubMed]

53. López-Rubio, A.; Suaza, J.D.; Porter, C.; Uribe, S.; Bedoya, G.; Vélez, I.D. Phylogenetic signal at the Cytb-SertRNA-IG1-ND1 mitochondrial region in Anopheles (Kerteszia) neivai Howard, Dyar \& Knab, 1913. Biomedica 2017, 37, 143-154. [PubMed]

54. Lorenz, C.; Patané, J.S.; Suesdek, L. Morphogenetic characterisation, date of divergence, and evolutionary relationships of malaria vectors Anopheles cruzii and Anopheles homunculus. Infect. Genet. Evol. 2015, 35, 144-152. [CrossRef] [PubMed]

55. Ramirez, C.C.L.; Dessen, E.M.B. The polytene chromosomes of the mosquito Anopheles bellator compared with those of Anopheles cruzii. Braz. J. Genet. 1996, 19, 555-558. [CrossRef]

56. Rona, L.D.; Carvalho-Pinto, C.J.; Gentile, C.; Grisard, E.C.; Peixoto, A.A. Assessing the molecular divergence between Anopheles (Kerteszia) cruzii populations from Brazil using the timeless gene: Further evidence of a species complex. Malar. J. 2009, 8, 60. [CrossRef]

57. Rona, L.D.; Carvalho-Pinto, C.J.; Mazzoni, C.J.; Peixoto, A.A. Estimation of divergence time between two sibling species of the Anopheles (Kerteszia) cruzii complex using a multilocus approach. BMC Evol. Biol. 2010, 10, 91. [CrossRef]

58. Rona, L.D.; Carvalho-Pinto, C.J.; Peixoto, A.A. Evidence for the occurrence of two sympatric sibling species within the Anopheles (Kerteszia) cruzii complex in southeast Brazil and the detection of asymmetric introgression between them using a multilocus analysis. BMC Evol. Biol. 2013, 13, 207. [CrossRef]

59. Rona, L.D.; Carvalho-Pinto, C.J.; Peixoto, A.A. Molecular evidence for the occurrence of a new sibling species within the Anopheles (Kerteszia) cruzii complex in south-east Brazil. Malar. J. 2010, 9, 33. [CrossRef] [PubMed]

60. de Rezende Dias, G.; Fujii, T.T.S.; Fogel, B.F.; Lourenço-de-Oliveira, R.; Silva-do-Nascimento, T.F.; Pitaluga, A.N.; Carvalho-Pinto, C.J.; Carvalho, A.B.; Peixoto, A.A.; Rona, L.D.P. Cryptic diversity in an Atlantic Forest malaria vector from the mountains of South-East Brazil. Parasit. Vectors 2018, 11, 36. [CrossRef] [PubMed]

(C) 2020 by the authors. Licensee MDPI, Basel, Switzerland. This article is an open access article distributed under the terms and conditions of the Creative Commons Attribution (CC BY) license (http://creativecommons.org/licenses/by/4.0/). 